\title{
NDE FOR CHARACTERIZING OXIDATION DAMAGE IN REINFORCED CARBON-CARBON
}

Don J. Roth*

National Aeronautics and Space Administration

Glenn Research Center

Cleveland, Ohio, United States

Richard W. Rauser

University of Toledo

Toledo, Ohio, United States

Nathan S. Jacobson

National Aeronautics and Space Administration

Glenn Research Center

Cleveland, Ohio, United States

Russell A. Wincheski

National Aeronautics and Space Administration

Langley Research Center

Hampton, Virginia, United States

James L. Walker

National Aeronautics and Space Administration

Marshall Space Flight Center

Marshall Space Flight Center, Alabama, United States

Laura A. Cosgriff

Cleveland State University

Cleveland, Ohio, United States

\section{ABSTRACT}

In this study, coated reinforced carbon-carbon (RCC) samples of similar structure and composition as that from the NASA space shuttle orbiter's thermal protection system were fabricated with slots in their coating simulating craze cracks. These specimens were used to study oxidation damage detection and characterization using NDE methods. These specimens were heat treated in air at 1143 and $1200{ }^{\circ} \mathrm{C}$ to create cavities in the carbon substrate underneath the coating as oxygen reacted with the carbon and resulted in its consumption. The cavities varied in diameter from approximately 1 to $3 \mathrm{~mm}$. Single-sided NDE methods were used since they might be practical for on-wing inspection, while x-ray micro-computed tomography (CT) was used to measure cavity sizes in order to validate oxidation models under development for carbon-carbon materials. An RCC sample having a naturallycracked coating and subsequent oxidation damage was also studied with $\mathrm{x}$-ray micro-CT. This effort is a follow-on study to one that characterized NDE methods for assessing oxidation damage in an RCC sample with drilled holes in the coating. The results of that study are briefly reviewed in this article as well. Additionally, a short discussion on the future role of simulation to aid in these studies is provided.

*Corresponding author: Phone: 216-433-6017, Fax: 216-977-7150, E-mail: donald.j.roth@nasa.gov 


\section{INTRODUCTION}

Reinforced carbon-carbon (RCC) with a silicon carbide coating for oxidation resistance is used on the NASA Space Shuttle Orbiter's wing leading edge, nose cap, and arrowhead attachment point to the external tank for thermal protection during re-entry. The strength and light weight of RCC make it an ideal aerospace material; however, oxidation is a major concern. Oxidation damage to RCC can occur if the silicon carbide coating is itself damaged but still intact such that hot gases have access to the carbon beneath the coating. ${ }^{1}$ In such cases, it is critical to evaluate the extent of the oxidation damage underneath the intact $\mathrm{SiC}$ coating. Even small breaches in the RCC coating system have recently been identified as potentially serious. In a prior study, small breaches in the coating of an RCC sample were created by drilling holes followed by oxidation of the sample and subsequent nondestructive (NDE) to characterize oxidation damage. ${ }^{2}$ In that study, RCC samples were oxidized to create approximately hemi-spherical holes underneath the silicon carbide coating and subsequently inspected using various nondestructive evaluation methods. In the current study, small breaches were created by machining slots of various widths to simulate cracks of various sizes in the coating, and NDE was again subsequently used to characterize the oxidation damage.

The NDE techniques employed in this study included state-of-the-art backscatter x-ray (BSX), ultrasonic guided waves, eddy current (EC), and thermographic methods. All of these methods are single-sided techniques thereby lending themselves to practical inspections of components only accessible from one side. Samples were also inspected with x-ray micro-computed tomography (CT) to evaluate the true dimensions and morphology of the holes, as well as natural crack formations. ${ }^{3}$ The controlled oxidation damage provides standards for investigating the effectiveness of various nondestructive evaluation techniques for detecting and sizing oxidation damage in this material. NASA Glenn Research Center led this investigation that had some of the top NDE specialists/facilities from NASA Glenn, NASA Langley, and NASA Marshall inspect these samples with the various NDE methods. The results of this study are also discussed in reference 4.

\section{EXPERIMENTAL}

RCC Material

Figure 1 is a schematic of reinforced carbon/carbon (RCC) with a Silicon Carbide (SiC) conversion coating. Briefly, this material is made with a two-dimensional layup of carbon-carbon fabric with repeated applications of a liquid carbon precursor to fill voids. An oxidation protection system is based on a $\mathrm{SiC}$ conversion coating. Because of the difference in coefficient of thermal expansion (CTE) of the $\mathrm{SiC}$ coating and carbon/carbon substrate, the $\mathrm{SiC}$ coating shrinks more than the underlying carbon/carbon on cooldown from the coating application temperature. This leads to vertical cracks in the coating, and these cracks are pathways for oxygen to reach the carbon/carbon substrate. Actual RCC used on the Space Shuttle Orbiter is infiltrated with tetraethyl orthosilicate (TEOS) which decomposes on a mild heat treatment to silica. Then the RCC surfaces are painted with a sodium silicate glass, which melts and seals cracks on re-entry. All the samples used in this study had the TEOS treatment. In addition, one of the samples studied had the sodium silicate glass. The samples were all approximately $5 \mathrm{~mm}$ thick.

The sample with SiC plus glass coating ( $\mathrm{RCC} 1)$ was a flat plate with an approximately $1 \mathrm{~mm}$ thick $\mathrm{SiC}$ plus glass coating and coated on all sides. The plate had an artificial craze crack of linear geometry made with a diamond blade (Keen Kut Products, Hayward, CA) of $0.25 \mathrm{~mm}$ thickness. This plate was used for ultrasonic studies. The slot was cut to the SiC plus glass coating/carbon-carbon interface on one side of the sample. 


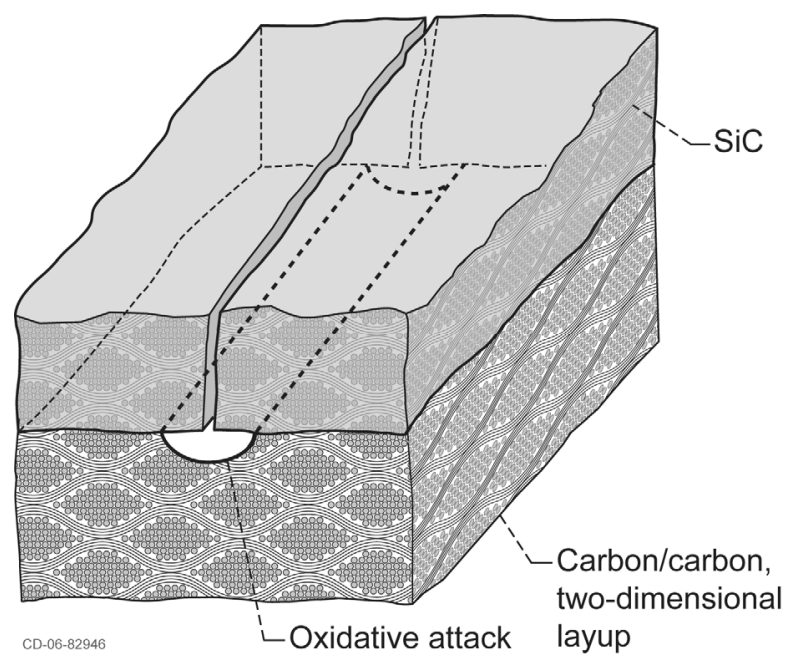

Figure 1. Schematic of SiC-protected carbon/carbon used in this study. Reprinted with permission from Elsevier.
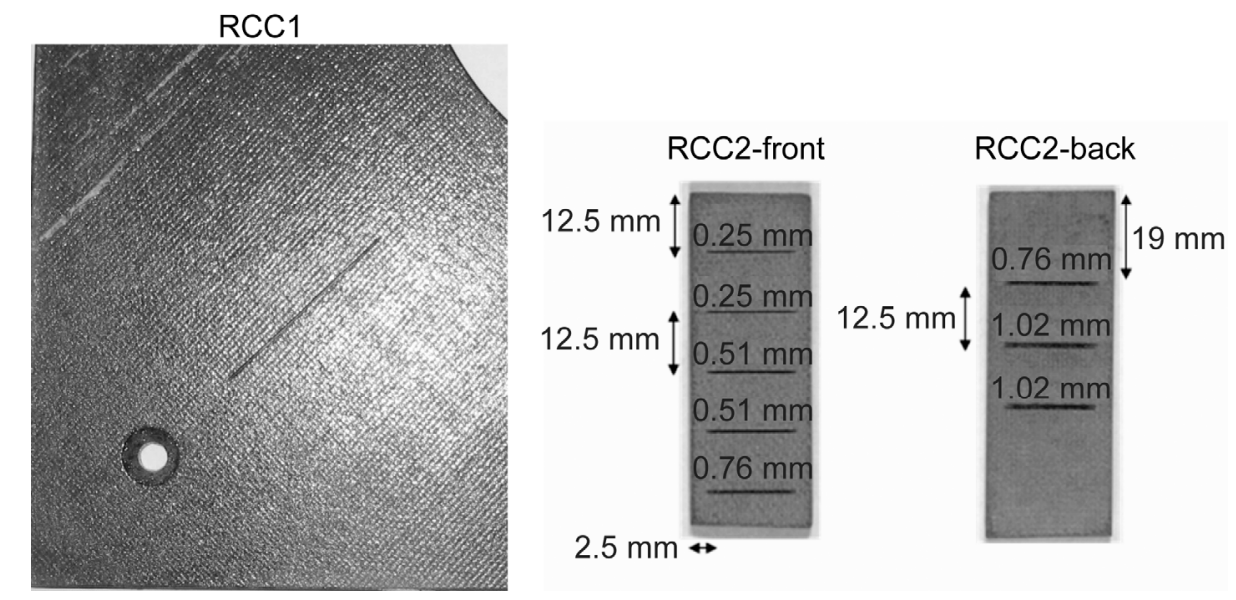

Figure 2. Photographs of machined RCC samples with machined slots. Nominal widths of slots are shown above the slot and spacing between slots and from edges is also shown.

The samples with only the $\mathrm{SiC}$ coating had an approximately $1 \mathrm{~mm}$ thick coating and were coated on all sides. Of these, one was a plate. The plate (RCC2) had machined slots made with diamond blades of $0.25,0.51,0.76$, and $1.02 \mathrm{~mm}$ thicknesses. These slots were cut to the $\mathrm{SiC} /$ carbon-carbon interface on both sides of the plate sample. These slotted specimens are shown in figure 2 .

Other SiC-only coated samples included several flat $1.91 \mathrm{~cm}$ diameter and $1.52 \mathrm{~cm}$ thick disks. ${ }^{3}$ Some of these had slots machined in them; others were used in their as-fabricated form with the naturally-occurring craze cracks acting as paths for oxidation. Polishing $\sim 300 \mu \mathrm{m}$ of $\mathrm{SiC}$ off the surface revealed the cellular crack pattern as shown in figure 3(a) together with a 'skeleton' trace of the cracks in figure $3(\mathrm{~b})$. 

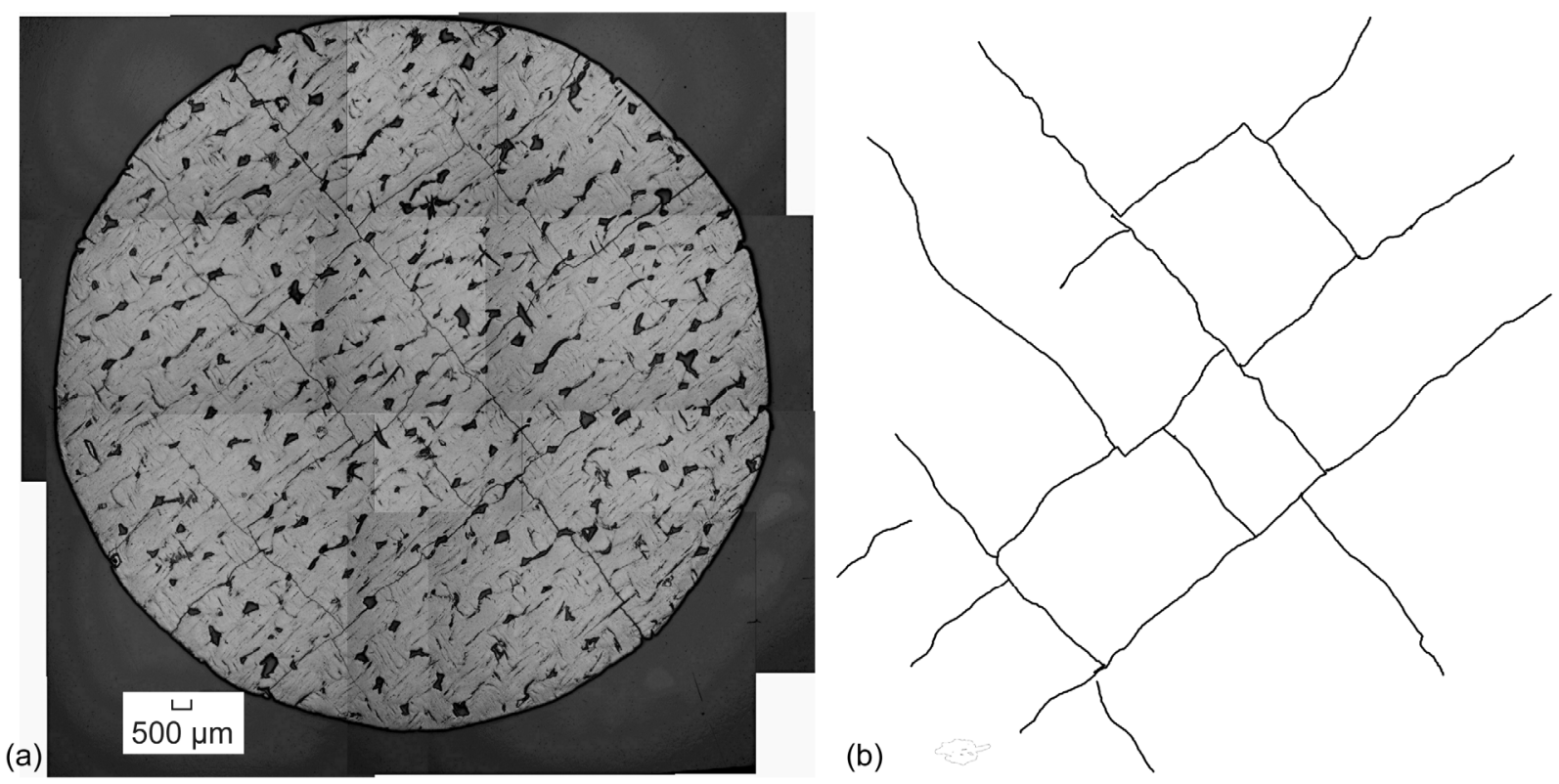

Figure 3(a) RCC disk with craze crack pattern on the surface. (b) 'Skeleton' trace of crack pattern. Reprinted with permission form Elsevier.
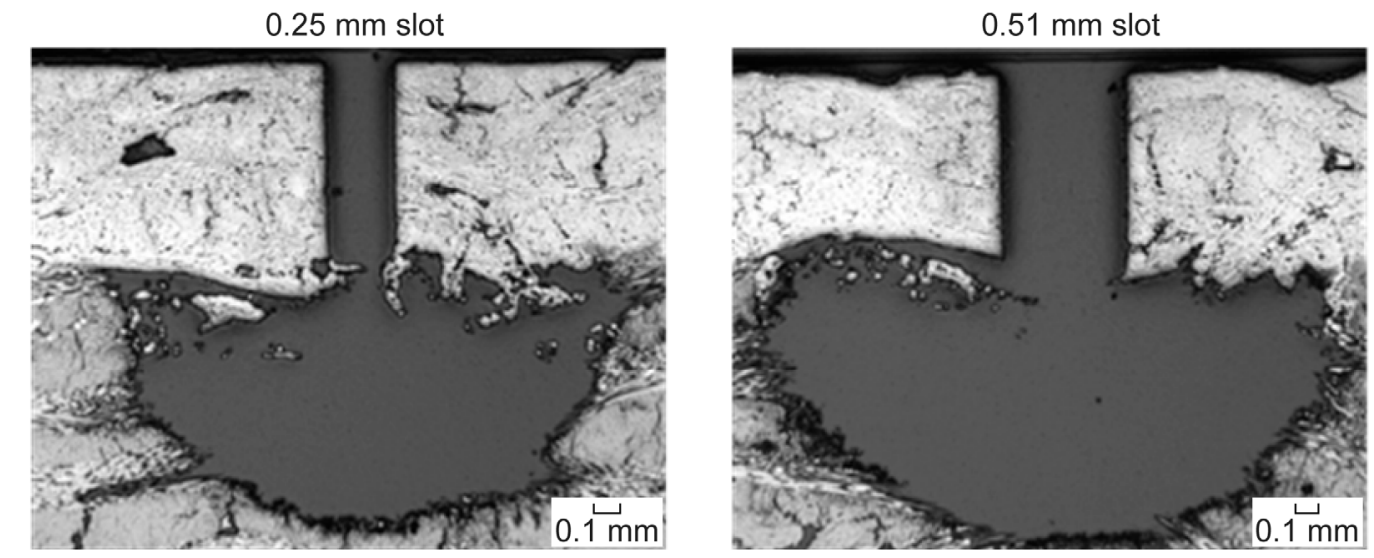

Figure 4. Cross-sections of cavity resulting from oxidation treatment for the slotted disks with an oxidation treatment for $0.5 \mathrm{hr}$ in $1200^{\circ} \mathrm{C}$ static laboratory air.

Controlled laboratory oxidation exposures were performed as follows: (a) Plates and disks with machined slots: $0.5 \mathrm{hr}$ at $1200{ }^{\circ} \mathrm{C}$ in static laboratory air; (b) Disk with craze cracks only: $2.5 \mathrm{hr}$ in bottle flowing air at $1143{ }^{\circ} \mathrm{C}$. The carbon/carbon substrate below the machined slot oxidized to form an approximate hemispherical void (fig. 4). This uniform attack pattern indicates diffusion control; and reference 3 describes an oxidation model for given slot and crack breaches in the coating. The resulting size of the void would be expected to increase with increasing slot width. Depth and diameter of voids in this study tended to be on the order of 1 to $3 \mathrm{~mm}$. This controlled oxidation damage provides standards for investigating the effectiveness of various nondestructive evaluation techniques for detecting and sizing this type of oxidation damage in this material. 
Nondestructive Evaluation (NDE)

Various single-sided state-of-the-art NDE methods were used to characterize the RCC samples prior to (baseline condition), and after, oxidation. These methods included backscatter x-ray (BSX), eddy current (EC), thermography, and guided wave ultrasonics. X-ray micro-computed tomography (CT) was used to size oxidation damage and cracking, and develop three-dimensional volumetric visualizations. References 5 to 9 provide basic principles for the various methods, and experimental parameters for the methods are given here. Specialized image processing was employed as needed to highlight indications from the NDE methods using software developed at NASA and available in the public domain. ${ }^{10}$ The software processing generally consisted of the following operations (in the following sequence): image crop, contrast expansion, outlier (bad value) removal, wavelet denoise. If the processing was applied, it was applied identically to the images of pre- and post-oxidation NDE images.

\section{Backscatter X-ray}

A scanning backscatter X-ray (BSX) system was used to image the RCC samples. ${ }^{5}$ Several parameter settings were employed to determine optimized conditions but for the purposes of comparing prior and post-oxidation images, the following settings were used in those cases. The settings were aperture of $2 \mathrm{~mm}$, voltage of $55 \mathrm{kV}$, current of $11.6 \mathrm{~mA}$, focal spot size of $1 \mathrm{~mm}$, finned collimator angle of $90^{\circ}$ and exposure time per scan position of $0.2 \mathrm{sec}$. The scan parameters included scan (X) and step (Y) increments of $0.5 \mathrm{~mm}$ and scan velocity of $2.5 \mathrm{~mm} / \mathrm{sec}$. Scans were performed from both sides of the RCC2 sample.

\section{Eddy Current}

Scanning eddy current (EC) inspection of the RCC sample was accomplished using a high frequency eddy current surface probe connected through a spring loaded z-axis gimbal to an $\mathrm{x} / \mathrm{y}$ scanning system. In the present work a probe drive frequency of $5 \mathrm{MHz}$ and scan spacing of $0.025 \mathrm{in}$. were used. Calibration of the system is performed using an uncoated RCC test article of nominally matching conductivity to the part under test. The probe response is rotated such that lift-off is in the horizontal direction. Nonconductive plastic shims are then used to measure the nominal SiC coating thickness (lift-off) and lift-off sensitivity. Oxidation damage under the $\mathrm{SiC}$ coating is measured as a localized increase in lift-off due to the increased spacing between the sensor and the conducting carbon-carbon substrate in the oxidized areas. Scans were performed from both sides of the sample. The differences in conductivity/lift-off values are displayed in various shades of gray in the c-scan image. The system used in this study minimized edge effects as compared to the one employed in reference 2 .

\section{Thermography}

A pulsed full-field thermographic NDE method utilizing flash lamps and a high speed camera was used to obtain images of the RCC sample. ${ }^{7}$ The system consists of two high energy xenon flash lamps, each capable of producing a $1.8 \mathrm{~kJ}$ flash with a $5 \mathrm{msec}$ duration. The flash lamps were placed at locations that provide a relatively uniform distribution of heat across the surface of the specimen. The transient thermal response of the specimen after flashing was captured using high-speed infrared camera. The camera used in the study is a 640 by $512 \mathrm{InSb}$ focal plane array type with a 14 bit dynamic range. The camera operates in the 3 to $5 \mu \mathrm{m}$ wavelength range and is capable of capturing thermal data at rates of $30 \mathrm{~Hz}$ for the full array size. For this study, a 320 by 256 portion of the full array was utilized in order to increase the frame rate to approximately $60 \mathrm{~Hz}$. Flash initiation, data collection, storage and processing were all performed using software on the acquisition computer. Experimental data was collected using the following procedure. The specimen was placed in front of 
the IR camera at a distance that allowed the sample to fill most of the active focal plane and then focused. Flash lamps were set at a distance of approximately $300 \mathrm{~mm}$. from the sample at an angle of $45^{\circ}$. Along with the images captured after the flash, 6 preflash images were collected. Instantaneous and derivative images (from relative temperature vs. time) were obtained, and the operator normally selected the best images for analysis using a subjective process of selecting frames of maximum contrast. Thermography was performed from both sides of the sample. The differences in surface temperature are displayed in various shades of gray in the image.

\section{Ultrasonic Guided Waves}

An ultrasonic guided wave measurement system $^{8}$ was employed to determine whether total ultrasonic energy $\left(M_{0}\right)$ of the time domain guided waveform was altered by the addition of the slot (artificial crack) and oxidation of the RCC1 sample containing the single slot. $M_{0}$ is calculated from the area under the curve of the power spectral density $S(f)$ of the time domain waveform according to:

$$
M_{0}=\int_{f_{\text {low }}}^{f_{\text {high }}} S(f) d f
$$

where $f_{\text {low }}$ and $f_{\text {high }}$ are the lower and upper frequency $(f)$ bounds of the integration range, respectively. Total energy is a physically understandable parameter that would likely be altered due to ultrasonic scattering both by the addition of the slot into the ultrasonic path and after further alterations of the slot due to oxidation and glass filling. Broadband ultrasonic transducers were used with center frequencies of approximately $1 \mathrm{MHz}$ (both sender and receiver were of the same frequency). Multiple mode excitation is likely due to the use of broadband transducers and the existence of multiple plate wave modes is confirmed by the complicated nature of the signal. ${ }^{8}$ Ultrasound was coupled to the material via elastic coupling pads. The distance between sending and receiving transducers was $2.5 \mathrm{~cm}$. For the baseline (prior to slotting) condition of the RCC1 sample (fig. 2), the transducers were positioned so that they would straddle the future position of the slot, and then after slotting, they were positioned identically such that they straddled the slot.

Analog-to-digital sampling rate for the ultrasonic testing was $10 \mathrm{MHz}$. A measurement was made $($ contact load $=3.63+0.23 \mathrm{~kg}[8+0.5 \mathrm{lb}])$, the sender-receiver pair was lifted, moved to the next location, lowered to be in contact with the sample, and another measurement made. 20 measurements were obtained ( 2 columns by 10 rows) with measurements separated by $1 \mathrm{~mm}$, and mean and standard deviation of $\left(M_{0}\right)$ was calculated. The identical pattern of measurements was made prior to slotting, after slotting, after oxidation, and after removing glass sealant from the crack followed by a second oxidation. Additionally, the final scan was done 5 times to measure reproducibility of the technique. In a future investigation, the effect of the slot and oxidation on other ultrasonic parameters that can be derived from broadband ultrasonic guided wave signals will be considered. ${ }^{8,10}$

\section{X-ray Computed Tomography}

X-ray computed tomography $(\mathrm{CT})$ was used to provide additional images of the oxidation damage and study oxidation in the naturally-cracked RCC sample, without destructive sectioning. ${ }^{9}$ This SmartScan Model 100 (CITA Systems, Inc., Pueblo, CO) system utilizes a Feinfocus FXE-160 (COMET AG, Flamatt, Switzerland) microfocus x-ray source to produce very high resolution imaging of samples, approaching $0.025 \mathrm{~mm}$, in the CT mode of operation. The major hardware components of this system included the x-ray source, an area detector system, a five-axis object positioning subassembly, and a lead-lined radiation cabinet. A dual-processor computer system controlled the data acquisition and image processing. The slice plane thickness was $0.120 \mathrm{~mm}$ per slice for these samples. Putting together slices electronically gave a three-dimensional view of oxidation damage. The sample was placed on a micropositioner between the source and detector which allowed positioning and 
rotation to obtain the slice images. The differences in x-ray density are displayed in various shades of gray.

\section{RESULTS AND DISCUSSION}

Figures 5 to 9 show NDE results including images of the samples prior to and after oxidation. Figure 5 shows backscatter x-ray results. Prior to oxidation, indications of the slots were difficult to discriminate when the x-ray source faced the RCC2 sample face with five slots (front) except for the bottommost slot which was approximately $0.76 \mathrm{~mm}$ wide (the widest of the five slots on that face). When the x-ray source faced the sample face having with three slots (back), those three slots could be discriminated fairly easily due to their large width $(0.76$ and $1.02 \mathrm{~mm})$ prior to oxidation. Post oxidation, all eight slots were easily discriminated with the x-ray source facing the front face indicating the extensive depth of damage created by the oxidation. A post-oxidation bsx image with x-ray source facing the back face is not shown (nor necessary) since all slots were revealed with $\mathrm{x}$-ray source facing the front face post-oxidation.

Figure 6 shows eddy current results. Slots were impossible to discriminate prior to oxidation but were easily discriminated after oxidation. Eddy current required scanning from both sides to allow visualization of each face's slots. Qualitatively, the larger slots appeared to give more pronounced indications.
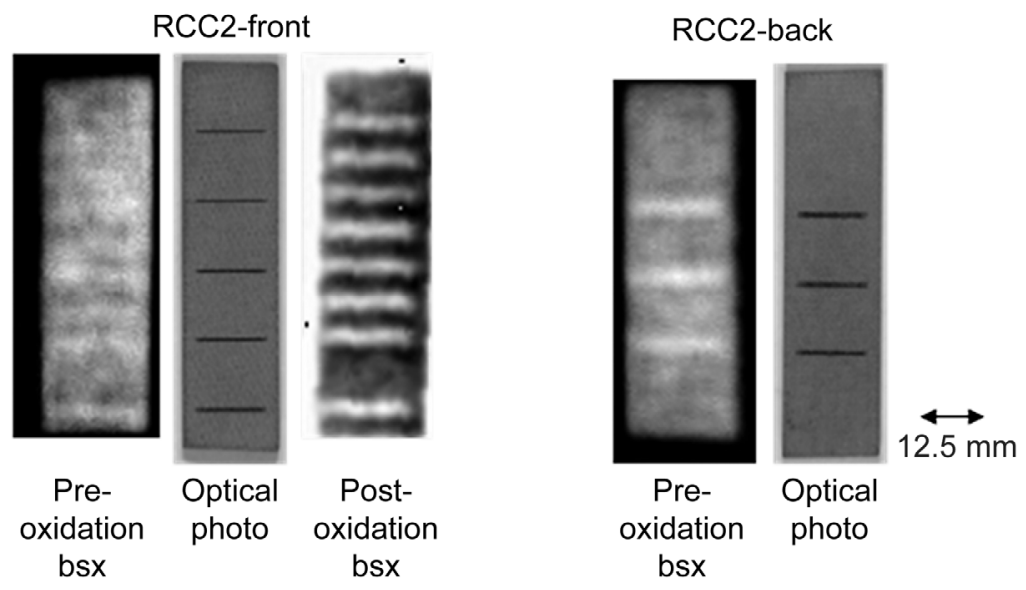

Figure 5. Backscatter x-ray results.
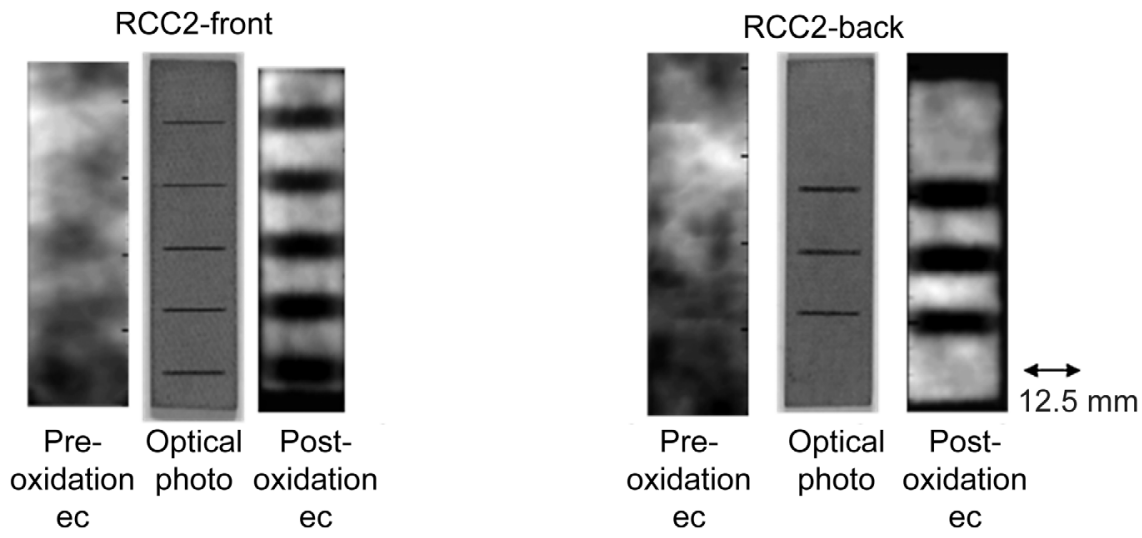

Figure 6. Eddy Current results.

Figure 7 shows thermography (derivative image) results. Slots were modestly discriminated prior to oxidation and were easily discriminated after oxidation. The heat source and thermography camera were facing the slots in order for oxidation damage detection to occur. 
Figure 8 shows ultrasonic guided wave (mean total energy $M_{0}$ ) results for RCC1 with the single machined slot. The guided wave technique is extremely sensitive to surface condition and the optical appearance of the surface appeared the same both before (baseline) and after oxidation. Controlled, identical force was present on the ultrasonic transducers for the ultrasonic measurements prior to and after oxidation. As compared to mean $M_{0}$ of the time domain wave for the baseline condition (prior to machining slot), mean $M_{0}$ had decreased after machining, after first oxidation, and after machining out glass in the slot and performing a second oxidation. However, the decrease was not monotonic for these steps. The general decrease in $M_{0}$ after slotting and subsequent oxidation treatments is indicative of the slot structure scattering the ultrasound such that less ultrasonic energy reaches than receiving transducer as compared to the baseline (no slot) condition. Note that the repeatability of the measurement method was good. The five scans that were repeated after the final step show variability of mean $M_{0}$ to be about 2 percent which is significantly less than the 8 percent decrease in $M_{0}$ noted from baseline to after slot machined condition. This variability is dependent upon surface condition and measurement load remaining very similar.
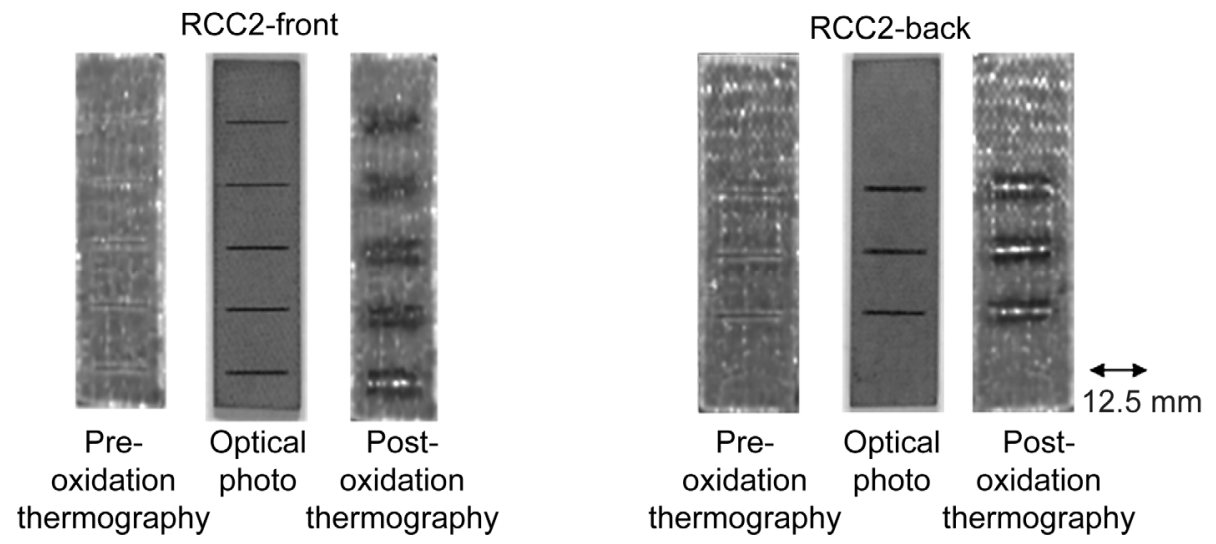

Figure 7. Thermographic (derivative) image results. Pre-oxidation images are frames at $0.18 \mathrm{sec}$ in the temperature versus time cooldown stream of frames. Post-oxidation images are frames at $0.164 \mathrm{sec}$ in the temperature versus time cooldown stream of frames.

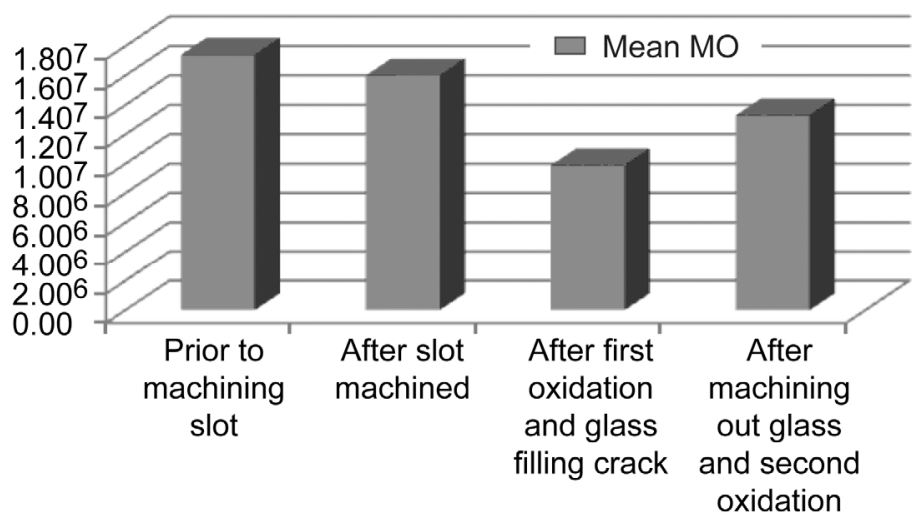

Figure 8. Ultrasonic guided wave (mean total energy $M_{0}$ ) results on sample RCC1 (fig. 2). Measurement variability is about 2 percent based on repeated trials. 


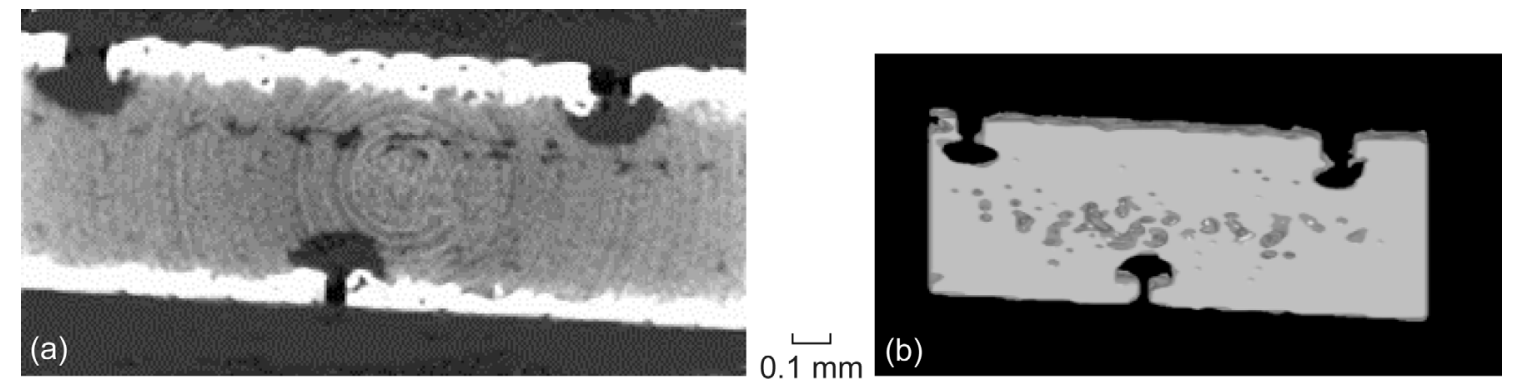

Figure 9. (a) Contrast-enhanced x-ray CT slice of portion of RCC2 sample. Ring pattern is an artifact of the CT processing. (b) Solid three-dimensional visualization constructed from CT slices.

Figure 9(a) shows an x-ray CT slice of a portion of the RCC2 sample showing the $0.25,0.51$, and $1.02 \mathrm{~mm}$ slots and the hemi-spherical oxidation damage beneath the $\mathrm{SiC}$ coating and slots. CT has been used to size the hemispherical regions in order to help validate and/or reveal deviations from the oxidation model discussed in references 1 and 3 . The size of the voids revealed by CT was on the order of 1 to $3 \mathrm{~mm}$ for depth and diameter, depending on the slot width. CT sizing was generally within 5 to 10 percent of actual values from destructive sectioning of several of the sample portions. X-ray CT allows the ability to sweep through various cross-sections and find the maximum diameter nondestructively and thus can result in a more accurate value for void diameter than destructive sectioning. Three-dimensional visualizations and animations composed of 11 consecutive CT slices were prepared on different sections of test samples, with one solid visualization shown in figure 9(b). These help reveal additional morphological features of the damage due to the three-dimensional nature.

The high resolution of the $\mathrm{x}$-ray CT technique makes it suitable for probing the oxidation damage below craze cracks, as this damage tends to be smaller and much more irregular than the damage below the machined slots. Figure 10 shows two x-ray CT slices for a naturally-cracked RCC sample with the outlined area indicating the oxidation damage below the natural crack paths. The oxidation cavity shapes are highly irregular, as expected from the varying coating thickness and irregular nature of the cracks. ${ }^{3}$ Figure 11 shows a translucent three-dimensional visualization constructed from 11 consecutive $\mathrm{CT}$ slices of this sample. 


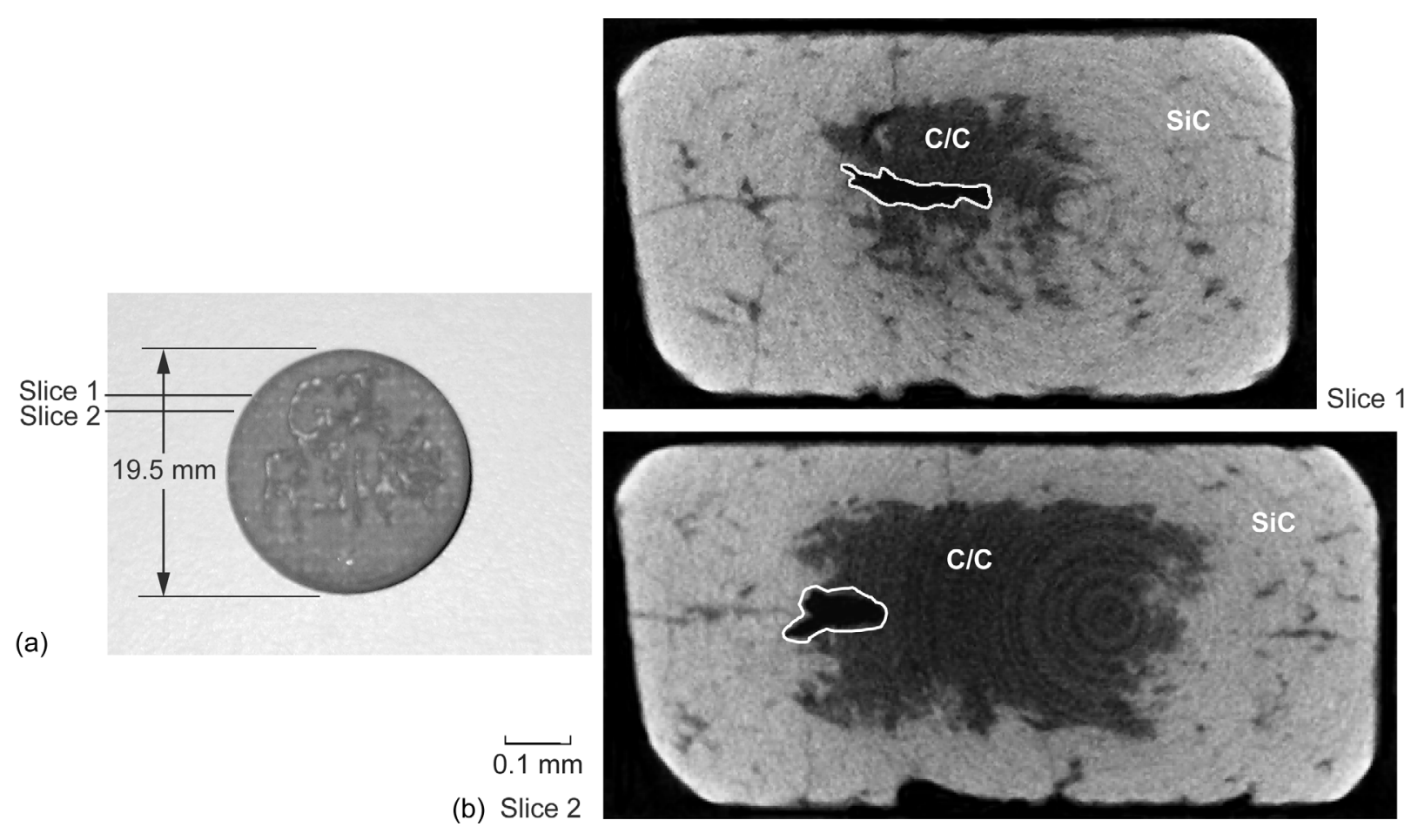

Figure 10. X-ray CT of SiC-coated RCC oxidized for $2.5 \mathrm{hr}$. in air at $1143^{\circ} \mathrm{C}$. (a) Location of CT slices. (b) Two CT slices. Reprinted with permission from Elsevier.

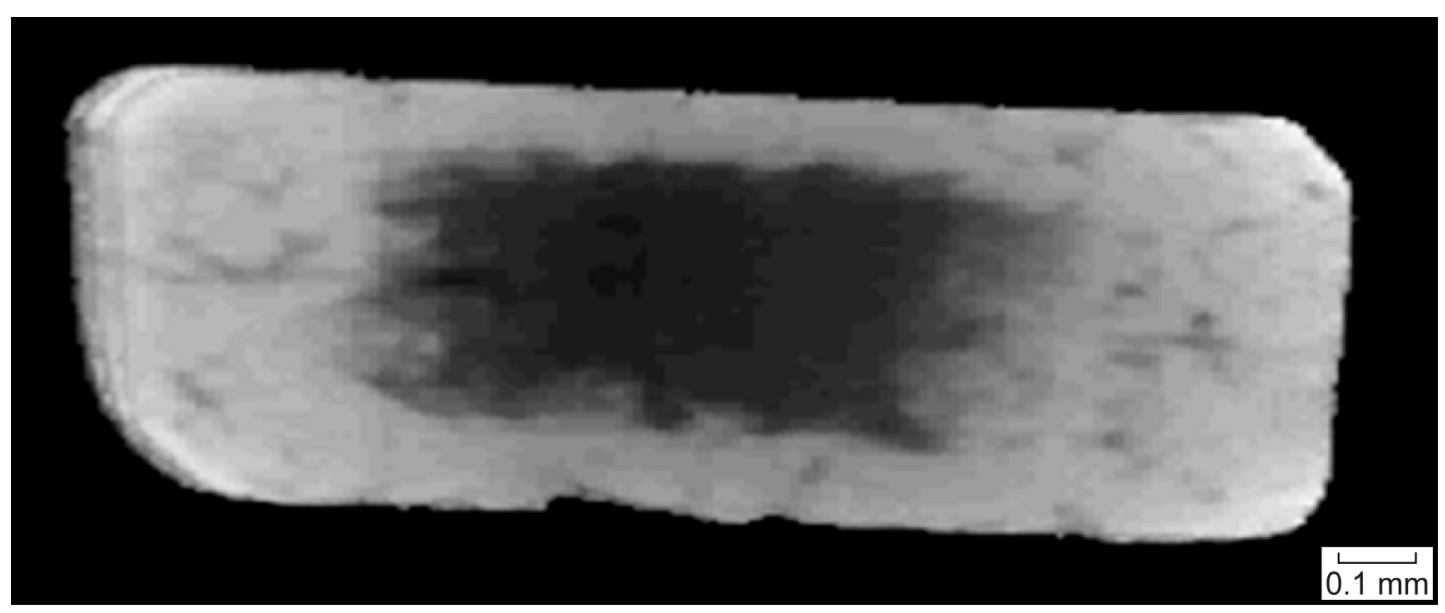

Figure 11. Translucent three-dimensional visualization constructed from X-ray CT slices of sample shown in figure 10 . 


\section{PRIOR STUDIES}

It is pertinent here to review the results of a prior related study in which a more quantitative approach to NDE capability was evaluated. ${ }^{2}$ In that study, RCC material that was the same as that used for the wing-leading-edge of the shuttle had holes rather than cracks incorporated to the depth of the coating in order to study oxidation damage with NDE methods. Seventeen $0.5 \mathrm{~mm}$ diameter holes, spaced $13 \mathrm{~mm}$ apart, were ultrasonically drilled through the coating system to the depth of the SiC coating, followed by oxidation treatments. The oxidation treatments were conducted in static (nonflowing) air at $1 \mathrm{~atm}$. and $1200{ }^{\circ} \mathrm{C}$ in a box furnace. A series of drillings followed by oxidation treatments was performed; first Batch 1 was drilled, then the disk was oxidized for 10 min, then Batch 2 was drilled, then the disk was oxidized for 10 min (giving 20 min on Batch 1 and 10 min on Batch 2 ), and so forth. The sample underwent seven drillings, and oxidation treatments varying from 10 to 70 min. The ability to measure the diameter and depth of resulting oxidation damage using NDE methods was evaluated. Figure 12 shows apparent diameter results from the various NDE methods and comparison to optically-measured diameters. Xray CT and thermography revealed the trend of void size increasing with increasing oxidation treatment time. Digital and Film Xray were not able to differentiate the void sizes. This is most likely due to the fact that the drilled hole and its extension into the cavity provided the dominant x-ray contrast over the oxidation damage. Figure 13 shows apparent depth of the holes beneath the coating from Xray CT and Eddy Current and their comparison to depth measured from optical measurements. Eddy current and CT measurements revealed the trend of void depth increasing with increasing oxidation treatment (with Xray CT providing the best results). Eddy current measurements will (and did) underestimate the actual void depth for small void diameters as unflawed material near the void will be averaged into the impedance measurement of the relatively large $(0.1$ by 0.05 in.) coils. Xray CT, the two-sided method, is the most accurate NDE method with regards to void sizing and measuring void depth.

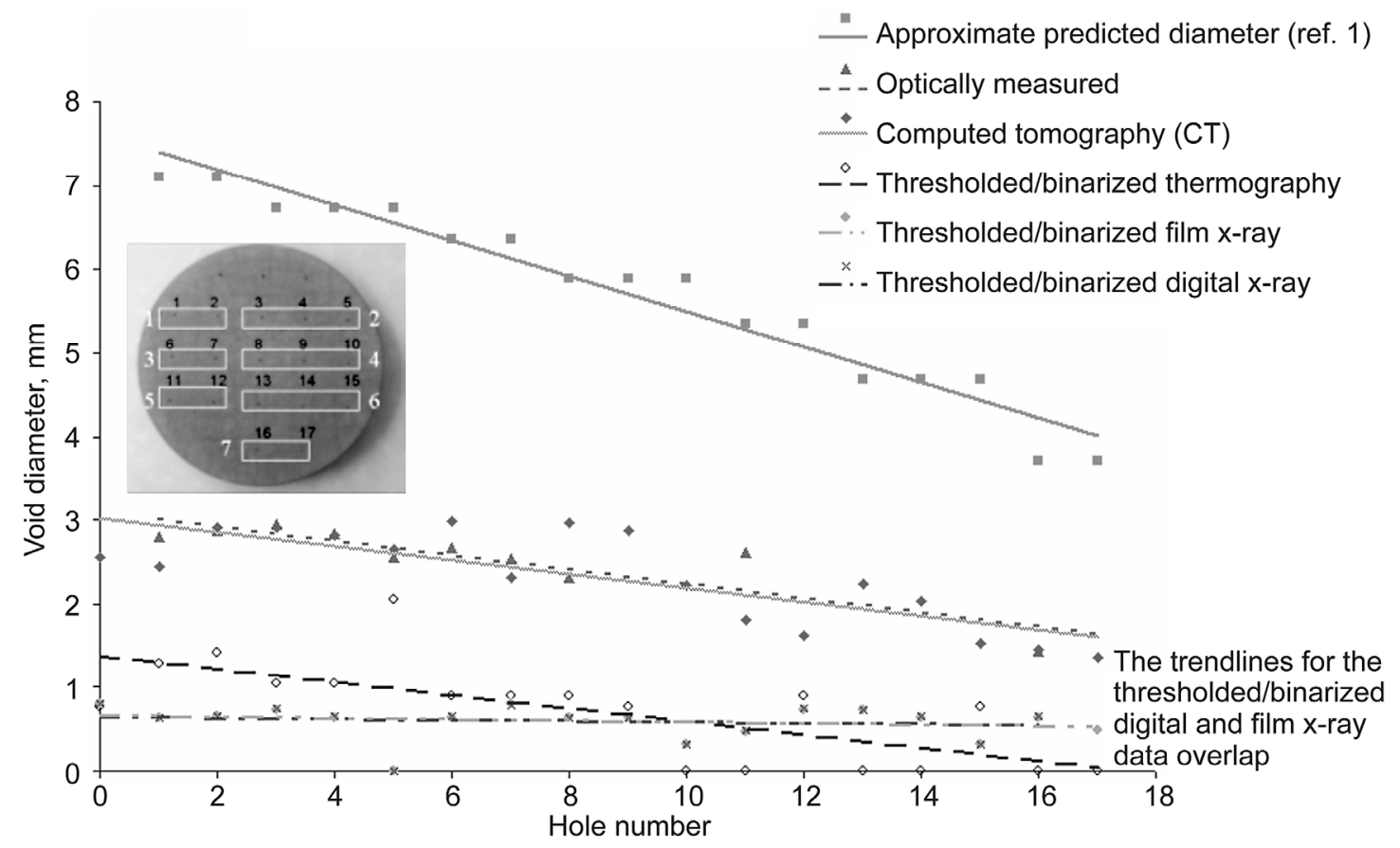

Figure 12. Apparent Void Diameter versus Hole Number from the different NDE methods and from optical measurement after sectioning. Disk schematic shows hole numbers (black) and oxidation batch numbers (white). Linear trend lines are shown. 


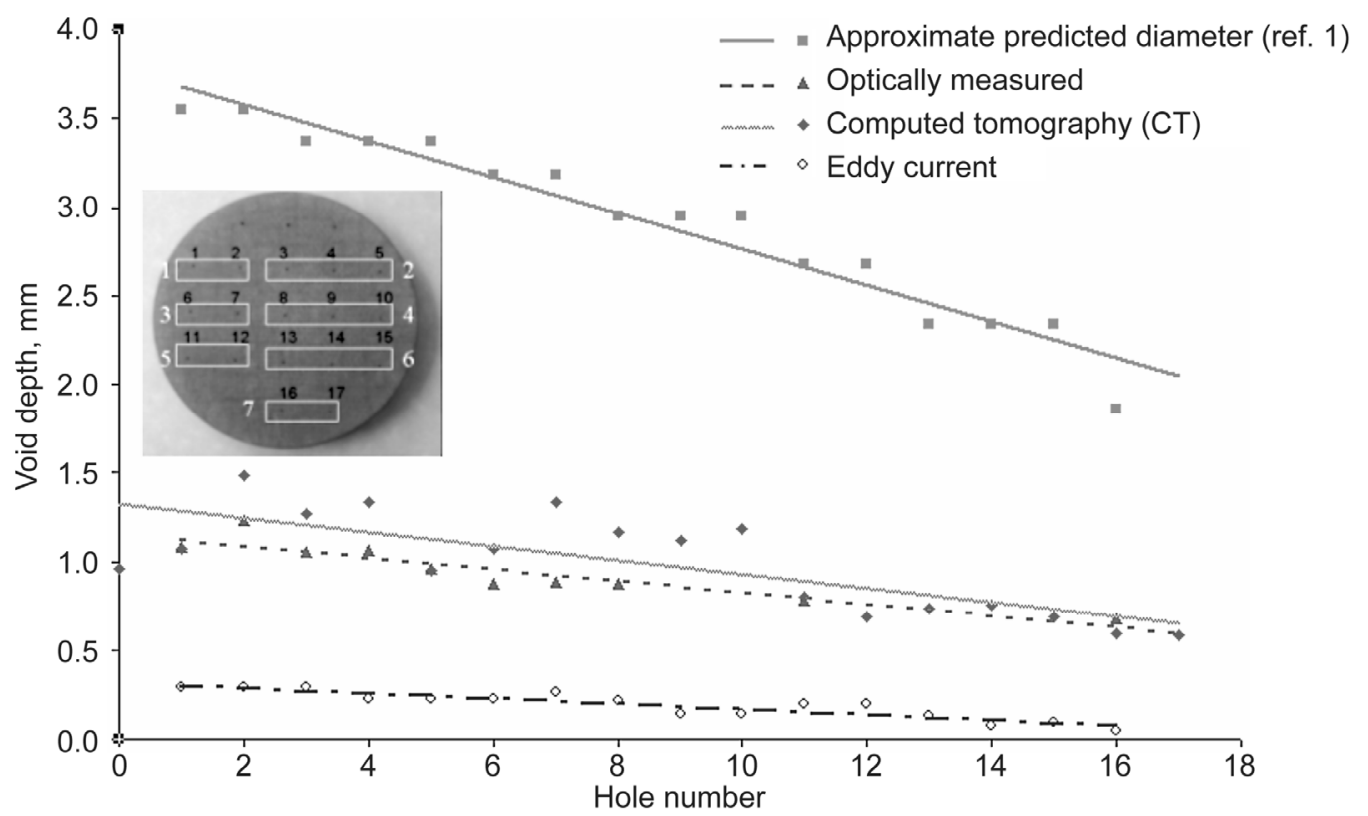

Figure 13. Apparent Void Depth versus Hole Number from different NDE methods and from optical measurement after sectioning. Disk schematic shows hole numbers (black) and oxidation batch numbers (white). Linear trend lines are shown.

\section{THE ROLE OF NDE SIMULATION STUDIES}

The ability to simulate NDE inspection scenarios is of great interest to NASA in order to quickly determine feasibility of specific inspection scenarios, design future inspections, and perhaps aid in the determination of the smallest detectable flaw size for a given inspection scenario (technique, material, geometry, access). Recently a NASA Engineering and Safety Center team of NDE experts engaged in an activity to evaluate existing inspection simulation tools and provide seed funds for development of usable simulation tools where physical models have been developed and at least partially validated. The team found that as with all simulation tools for physical phenomena, computational NDE tools represent an idealization of the true physical situation. Approximations are made with respect to geometry, material properties, measurement systems, underlying physical laws, and solution methods. A thorough understanding of these approximations, along with knowledge of the specific application requirements, is needed to develop appropriate and useful simulation results. Of pertinence to this particular study and that of reference 2 are nondestructive evaluation simulation methods for x-ray and x-ray computed tomography, thermography, eddy current and possibly ultrasonic guided wave inspection. In future studies, we will report on the use of some of these tools as applied to ceramic and 
composite structure inspection scenarios. Reference 11 provides an excellent introduction to the topic of NDE simulation.

\section{SUMMARY}

In this study, RCC samples having slots in their coating machined to the depth of the SiC coating, underwent oxidation treatments to create void-like damage in the carbon-carbon substrate underneath the coating. The size of the voids revealed by x-ray micro-CT was on the order of 1 to $3 \mathrm{~mm}$ for depth and diameter, depending on the slot width. State-of-the-art single-sided NDE methods, practical where access to only one side of the structure is available such as for on-wing inspections, were used to detect the damage. These methods included backscatter x-ray, eddy current, thermography, and ultrasonic guided waves. All of the methods were successful at detecting the oxidation damage, whereas only thermography unambiguously revealed the slots prior to oxidation. The higher resolution of the CT technique made it suitable to quantitatively assess oxidization damage both below machined slots and craze cracks. Further this technique revealed the patterns of oxidation attack, which tended to be smaller and much more irregular below the craze cracks than under the machined slots. Changes in ultrasonic guided wave total energy show this parameter is sensitive to structural changes in RCC. In prior studies, thermography revealed the trend of void size increasing with increasing oxidation treatment time, and eddy current revealed the trend of void depth increasing with increasing oxidation treatment. NDE simulation methods are expected to play a greater role in the future in assessing the feasibility of specific inspection scenarios and perhaps aid in the determination of the smallest detectable flaw size for a given inspection scenario (technique, material, geometry, access).

\section{REFERENCES}

${ }^{1}$ Jacobson, N.S., and Curry, D.M. , "Oxidation microstructure studies of reinforced carbon/carbon," Carbon 44 (2006) 1142-1150.

${ }^{2}$ Roth, D.J., Jacobson, N.S, Gray, J.N., Cosgriff, L.M., Bodis, J.R., Wincheski, R.A., Rauser, R.W., Burns, E.A., and McQuater, M.S., "NDE for Characterizing Oxidation Damage in Reinforced CarbonCarbon Used on The NASA Space Shuttle Thermal Protection System," Ceram. Eng. Sci. Proc., vol. 26, no. 2, 2006, pp. 133-141.

${ }^{3}$ Jacobson, N.S., Roth, D.J., Rauser, R.W., Cawley, J. D., and Curry, D.M., “Oxidation through coating cracks of SiC-protected carbon/carbon,” Surf. \& Coatings Tech. 203 (2008) 372-383.

${ }^{4}$ Roth, D.J., Rauser, R.W., Jacobson, N.S., Wincheski, R.A., Walker, J.L., Cosgriff, L.M., "NDE for Characterizing Oxidation Damage in Cracked Reinforced Carbon-Carbon," NASA TM-2009-215489 and accepted for publication in International Journal of Applied Ceramic Technology.

${ }^{5}$ Dugan, E., Jacobs, A., Shedlock, D., and Ekdahl, D., "Detection of Defects in Foam Thermal Insulation Using Lateral Migration Backscatter X-ray Radiography," Proceedings of SPIE 49th Annual Meeting, Symposium on Optical Science and Technology, Penetrating Radiation Systems and Applications VI, vol. 5541, Denver, August, 2004.

${ }^{6}$ Wincheski, B.A. and Simpson, J.W., "Application of Eddy Current Techniques for Orbiter Reinforced Carbon-Carbon Structural Health Monitoring," Contract\# 23-376-70-30-05, 2005.

${ }^{7}$ Shepard, S.M., Lhota, J.R., Rubadeux, B.A. and Ahmed, T., "Onward and Inward: Extending the Limits of Thermographic NDE,” Proc. SPIE Thermosense XXII, vol. 4020, 2000, p. 194.

${ }^{8}$ Roth, D.J., Cosgriff, L.M., Verilli, M.J., and Bhatt, R.T., "Microstructural and Discontinuity Characterization in Ceramic Composites Using an Ultrasonic Guided Wave Scan System," Materials Evaluation, vol. 62, no. 9, 2004, pp. 948-953.

${ }^{9} \mathrm{NDE}$ of advanced turbine engine components and materials by computed tomography, Yancey, R.N.; Baaklini, George Y.; Klima, Stanley J., ASME, International Gas Turbine and Aeroengine Congress and Exposition, 36th, Orlando, FL, June 3-6, 1991. 
${ }^{10}$ Roth, D.J., Martin, R.E., Seebo, J.P., Trinh, L.B., Walker, J.L., and Winfree, W.P., “A Software Platform for Post-Processing Waveform-Based NDE," SAMPE Proceedings and presentation, June 2007, Baltimore, MD.

${ }^{11}$ Aldrin, J.C. and Knopp, J.S., "Modeling and Simulation for Nondestructive Testing With Applications to Aerospace Structures,” Materials Evaluation, vol. 66, no. 1, 2008, pp. 53-59. 